\section{Keine Angst vor Ausdauersport bei Asthma}

\author{
Hochleistungssportler haben ein erhöhtes Risiko, an Asthma \\ bronchiale zu erkranken und Episoden mit Anstrengungsasthma zu \\ erleben. Bei Amateuren scheint dagegen ein moderates Ausdauer- \\ training die Atemwegsfunktion nicht negativ zu beeinflussen.
}

\begin{abstract}
_ orscher aus dem französischen Montpellier untersuchten 95 Männer, die in ihrer Freizeit seit im Mittel 8,5 Jahren jede Woche regelmäßig ein moderates Ausdauertraining absolvierten. Die Männer waren im Schnitt 32 Jahre alt, die Spanne reichte von 18 bis 67 Jahren. Die wöchentliche Trainingsdauer lag zwischen 2 und 20 Stunden, im Mittel betrug sie 10 Stunden. Von den 95 Probanden übten sich 40 im Triathlon, 37 im Radfahren, 15 im Langstreckenlauf und drei im Ausdauertrecking.

Nach der Erfassung von anamnestischen Daten zu bekannter Asthmadiagnose, vorbestehender Asthmasymptomatik und Anstrengungsasthma, Atemwegsallergien, aktueller Medikation und familiärer Vorbelastung mittels Frage-
\end{abstract}

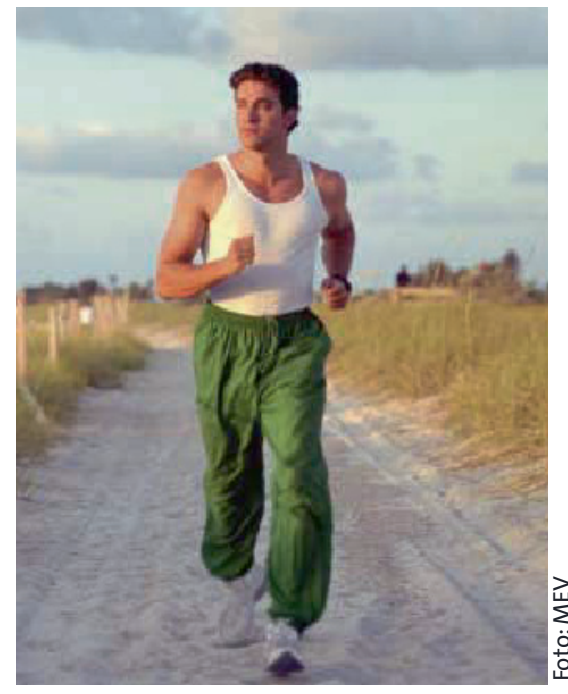

Ausdauersport - gut für Herz und Lunge bogen wurden alle Probanden einer Spirometrie unterzogen.

Keiner der Freizeitsportler wies zum Zeitpunkt der Untersuchung eine Obstruktion in Ruhe auf. Vier der 95 Athleten waren ärztlich diagnostizierte Asthmatiker. Diese Probanden und ein weiterer hatten auch bereits ein ärztlich dokumentiertes Anstrengungsasthma erlebt. Die Asthmaprävalenz in dieser Untersuchungsgruppe lag damit mit 4,2\% vergleichbar hoch wie in der südfranzösischen Referenzbevölkerung und deutlich niedriger als bei Leistungssportlern, für die in früheren Studien eine Asthmaprävalenz von $10-50 \%$ belegt worden ist.

Fazit: Ein regelmäßiges Ausdauertraining von 10 Stunden pro Woche in gemäßigtem Klima scheint das Risiko für die Entwicklung einer Atemwegserkrankung nicht zu erhöhen.

FK

Kippelen $\mathbf{P}$ et al. Asthma and exerciseinduced bronchoconstriction in amateur endurance-trained athlets. Int J Sports Med 2004; 25: 130-2

\title{
Interleukin-8-Polymorphismen bei Asthma und juveniler Arthritis
}

Das Zytokin Interleukin-8 ist ein wichtiger Mediator bei akuten und chronischen Entzündungen wie Asthma oder der juvenilen idiopathischen Arthritis, spielt aber auch bei viralen und bakteriellen Infektionen eine Rolle. Im Rahmen einer Studie wurde bei Patienten mit verschiedenen Erkrankungen nach IL-8-Polymorphismen gesucht, um genetische Grundlagen dieser Krankheiten zu finden.

Eorscher der Universität Freiburg anaIysierten die für IL-8 codierenden DNA-Bereiche von Kindern mit Asthma, Atopie oder einer juvenilen idiopathischen Arthritis auf die bekannten IL8-Polymorphismen -A251T, C781T, C1633T und A2767T hin und verglichen sie mit den entsprechenden DNAAbschnitten gesunder Kinder.

Alle IL-8-Polymorphismen waren mit dem Vorliegen von Asthma assoziiert (zwischen $\mathrm{p}=0,008$ und $\mathrm{p}=0,03$ ) - ein Befund, der jetzt erstmalig publiziert wurde. Kinder mit den-251T-und
781C-Allelen hatten signifikant häufiger Asthma als die gesunden Probanden, diese Varianten könnten damit einen Risikofaktor für die Asthmaentwicklung darstellen. In einer anderen Studie wurde gezeigt, dass das Vorliegen des -A251Allels zu einem erhöhten Risiko für eine Infektion mit Respiratory Syncytial Virus (RSV) und eine RSV-Bronchiolitis beiträgt - ein Hinweis darauf, dass diese Erkrankungen doch keine genetischen Gemeinsamkeiten mit Asthma haben, wie bisher vermutet wurde. Weiterhin fanden sich alle Polymorphismen häufi- ger bei Kindern mit Arthritis als bei Kindern mit Asthma. Die Assoziationsergebnisse lassen weiter den Schluss zu, dass gegenteilige Effekte der IL-8-Polymorphismen bei der chronisch juvenilen Arthritis und beim Asthma vorliegen.

Fazit: Bestimmte Polymorphismen bei den für IL-8 codierenden Genen sind mit dem Vorliegen von Asthma assoziiert. Asthma und die RSV-Bronchiolitis haben verschiedene genetische Hintergründe: Der Polymorphismus von IL-8, der mit einer Empfänglichkeit für eine RSV-Bronchiolitis assoziiert ist, könnte möglicherweise vor Asthma schützen. Eine im Vergleich zu Asthmakranken inverse Verteilung der Polymorphismen fand sich bei der juvenilen Arthritis. $b k$

Heinzmann A et al. Association study suggests opposite effects of polymorphisms within IL8 on bronchial asthma and respiratory syncytial virus bronchiolitis. J Allergy Clin Immunol 2004; 114: 671-6 